\title{
First Trimester Procedural Abortion in Family Medicine
}

Richard John Lyus, MBBS, BSc, Paul Gianutsos, MD, MPH, and Marji Gold, MD

Unintended pregnancy is common, and in the United States almost half of all women will have at least one abortion during their lifetime. The majority of abortions are performed in the first trimester. Although advances have been made in the provision of medical abortion in the family medicine setting, procedural methods remain the cornerstone of abortion care. We present a step-wise review of first trimester procedural abortion using the manual vacuum aspirator to demonstrate the feasibility of incorporating this service into a primary care setting. (J Am Board Fam Med 2009;22:169-174.)

In the United States, half of all pregnancies are unintended, and half of those will end in elective abortion. ${ }^{1}$ In 2005, 1.2 million abortions were performed in the United States, and at least one-third of women will have an abortion during their lifetime. Just under $90 \%$ of abortions are done before 12 weeks gestational age. ${ }^{1}$ Although there are variations in the definition of the first trimester, for this review gestations at 12 weeks from the last menstrual period or less will be considered the first trimester. Although most firsttrimester abortions are done in high-volume practices, a minority are performed in primary care physicians' offices, where this common procedure has been incorporated into the "basket of services" available. Abortion can be accomplished with medication or procedurally. Medical abortion is considered a treatment, not a procedure, because it involves counseling and the administration of medications to induce abortion. Although medical abortion has been shown to be safe and effective, most women eligible for medical abortion continue to choose procedural methods.

The early abortion procedure has been traditionally referred to as "surgical" abortion, but to both patients and providers this term suggests "cutting " in an operating room. More suitable terms are "procedural," "aspiration," or "suction" abor-

This article was externally peer reviewed.

Submitted 4 September 2007; revised 15 April 2008; accepted 18 April 2008.

From Swedish Family Medicine Cherry Hill, Seattle, WA (RJL, PG); and Albert Einstein College of Medicine and Montefiore Medical Center, New York, NY (MG).

Funding: none.

Conflict of interest: none declared.

Corresponding author: Richard John Lyus, 20 Timothy's Bridge Road, Stratford Upon Avon, Warwickshire, England CV379BF (E-mail: richard.lyus@swedish.org). tion, which better represent what is involved. ${ }^{2}$ Abortion during the first trimester is more accurately compared with endometrial biopsy or intrauterine device insertion than surgery. For many years, procedural abortion was accomplished using electric vacuum aspiration (EVA), which creates continuous suction using an electric-powered machine. Although EVA is still commonly used, manual vacuum aspiration (MVA) has for some providers replaced EVA for procedural abortion during the first trimester. MVA uses a hand-held, doublevalve plastic syringe (Figure 1); pulling back on the plunger creates a vacuum, which allows suction curettage to be performed when the valve is released. This hand-held device has major advantages compared with the electric suction machine: it is quiet, smaller, more portable, and less expensive. There are several reports of MVA abortion being successfully incorporated into family medicine practices and training, ${ }^{3-8}$ and most residents in these programs value integrated abortion training. ${ }^{9}$ In addition, one survey found that the majority of family medicine clinic patients who would consider using abortion services would prefer to receive them at their primary care clinic rather than visit a freestanding, high-volume abortion clinic. ${ }^{10}$

The steps involved in procedural abortion are (1) counseling and evaluation before the procedure, including selection of pain control methods; (2) offering appropriate analgesia and/or anesthesia; (3) achieving adequate cervical dilation; and (4) complete aspiration of uterine contents. If the clinician is certain that the abortion is complete and if the patient chooses to initiate her contraceptive method at that visit, no follow-up appointment is needed. ${ }^{11}$ 


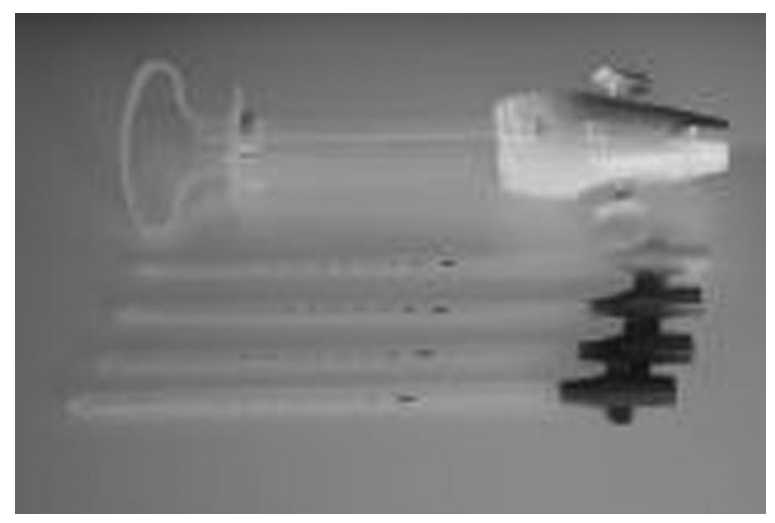

Figure 1. A manual vacuum aspiration device with cannulae. Picture is reprinted with permission of Ipas (www.ipas.org).

\section{Counseling}

Options counseling for unintended pregnancy is an Accreditation Council for Graduate Medical Education program requirement for residency training in Family Medicine. ${ }^{12}$ All family physicians must be competent to help patients consider abortion, adoption, and parenting, and to refer to appropriate services when necessary. Patients who choose abortion should be counseled regarding medical and procedural methods and the risks involved with each. Patients should be reassured that first trimester abortion does not increase the risk for subsequent adverse pregnancy outcomes; when abortion is safe and legal, their chances of getting pregnant and staying pregnant in the future are not affected by the abortion. There is no increase in rates of ectopic pregnancy, spontaneous abortion, preterm birth, or low birth weight, ${ }^{13,14}$ and no association with an increase in breast cancer risk. ${ }^{15}$

\section{Preprocedure Evaluation}

Before the procedure, the gestational age of the pregnancy must be determined to insure that the patient is eligible for an early abortion. The clinician should also determine whether the patient has any medical condition that precludes having an abortion in the family medicine setting. In addition, the patient's Rhesus blood type must be known and the appropriate dose of immune globulin given if she is Rhesus negative. No other laboratory testing is routinely necessary.

Because National Abortion Federation guidelines do not require the use of ultrasound for gestational dating, ${ }^{16}$ this may be determined by history of the last menstrual period and pelvic examination. However, pre-abortion sonography is the standard of care for most providers in the United States. The skills to perform ultrasound to date a pregnancy are easily learned and applicable to other areas of practice. We would encourage providers to use transabdominal ultrasound probes whenever possible because most first-trimester pregnancies can be seen with this approach and it is less invasive than the transvaginal approach.

First-trimester abortion is an extremely safe procedure and there are few contraindications. Any condition that might complicate a procedural abortion will almost certainly pose greater risk with continued pregnancy and childbirth. However, a comprehensive medical history should be taken to identify any chronic medical conditions or gynecologic problems. It is useful to know the details of past pregnancies, deliveries, and abortions; this history may affect the provider's approach to cervical preparation, pain control, or follow-up. Rarely, with medically complicated patients, it may be appropriate to perform the abortion in an inpatient setting where staff and facilities are readily available to manage complications.

\section{Pain Control}

Although some first-trimester procedural abortions take place using general anesthesia or intravenous sedation, $58 \%$ of providers surveyed in 2001 used only paracervical block with or without oral anxiolytics or analgesics. ${ }^{17}$ Because the discomfort experienced during abortion is a complex combination of physical and psychological factors, options for pain control should be discussed with the patient before the procedure. Most primary care providers will be able to offer a nonsteroidal anti-inflammatory drug before the procedure, an oral anxiolytic or narcotic analgesic, and paracervical block. Nonsteroidal anti-inflammatory drugs have been shown to reduce postprocedure pain ${ }^{18}$ and do not increase bleeding. Oral anxiolytics may be given, but have not been shown to significantly affect pain or anxiety scores. ${ }^{19}$ The use of preprocedure oral narcotics has not been studied. Attention to the woman's wellbeing and appropriate conversation can also increase her comfort during the procedure..$^{20,21}$

An abortion procedure is generally performed with the woman in the lithotomy position, using comfortable leg supports rather than foot rests if 
available. To perform the paracervical block, the speculum is placed inside the vagina to visualize the cervix, which may be cleansed with betadine or a similar solution. A small amount of local anesthetic is injected directly into the cervix at the 12- or 6-o'clock position to allow application of a tenaculum. The remainder of the local anesthetic is then injected into the tissue around the cervix. Only $22 \%$ of providers surveyed in 2001 used a 2 -site injection technique; the majority of providers inject at 4 sites or more. Although the exact site of the injections has not been shown to affect efficacy, deeper injections (inserting the needle $1 \frac{1 / 2}{2}$ inches) have been shown in one study to significantly reduce pain scores. ${ }^{22}$ It is prudent to aspirate slightly once the needle tip is in place to avoid intravascular injection.

\section{Cervical Priming and Dilation}

To permit passage of the cannula into the uterus, the cervix must be adequately dilated. The amount of dilation needed is generally determined by the gestational age of the pregnancy. There is some variation, but approximately half of providers use a cannula with a diameter equal in millimeters to the gestational age in weeks. ${ }^{17}$ For example, an 8 -week pregnancy would be aspirated through an 8 -mm cannula, and the cervix would therefore need to be dilated to $8 \mathrm{~mm}$. Other providers dilate to 1 or 2 millimeters more, and a minority dilate to a little less. ${ }^{17}$

\section{Mechanical Dilation}

For first-trimester abortion, cervical dilation is usually achieved using a series of rigid dilators, each dilator being slightly larger than the previous one. There are at least 4 different types of dilator, but the most frequently used are the gradually tapered Pratt and Denniston types (Figure 2). Although procedural abortion does not require sterile technique, providers should adhere to the "no-touch technique," where nothing that will be passed into the uterus touches anything unsterile beforehand. The dilators should therefore be held in the middle because the ends are passed through the internal cervical os.

\section{Cervical Priming}

Preparation of the cervix several hours before mechanical dilation is referred to as cervical priming and

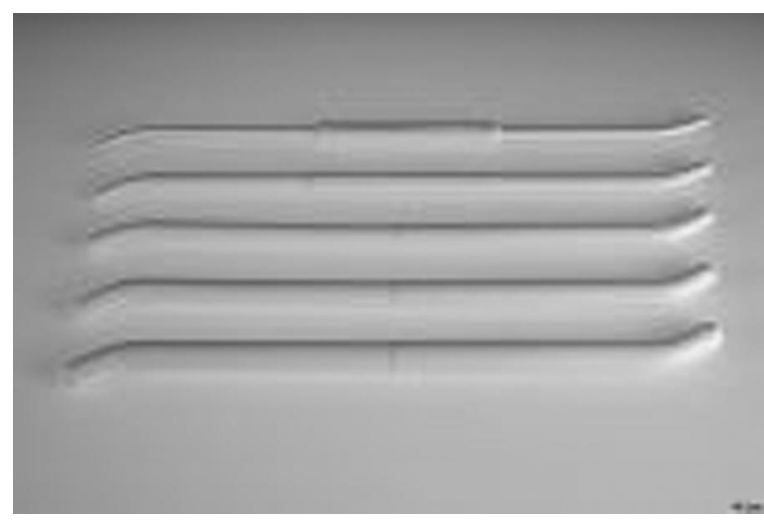

Figure 2. Denniston dilators of increasing size (top to bottom). Picture is reprinted with permission of Ipas (www.ipas.org).

is performed with medication and/or osmotic dilators. Priming may provide adequate dilation and avoid the use of mechanical dilators, or make the process of mechanical dilation easier. Osmotic dilators are short, water-absorbent rods placed in the cervical canal several hours before the procedure. They work by slowly expanding to dilate the canal with radial force, or through a biochemical softening of the cervix. Only $2 \%$ of providers use osmotic dilators routinely for first-trimester procedural abortion before 12 weeks gestation. ${ }^{17} \mathrm{~A}$ major drawback is the need for an additional procedure to place the dilators at least 2 to 4 hours before aspiration, and a place for the woman to wait while the priming occurs.

Misoprostol can be used as a pharmacologic agent for cervical priming. Misoprostol is an inexpensive, temperature-stable prostaglandin analog originally approved for gastric protection but now commonly used for cervical ripening in labor induction. Administered vaginally, orally, or sublingually 2 to 4 hours before the procedure, misoprostol has been shown to dilate the cervix, ease mechanical dilation, reduce the force required for dilation, reduce procedure time, and reduce estimated blood loss. ${ }^{23}$ However, none of these outcomes are likely to be clinically significant in the first trimester. In addition, misoprostol may cause cramping, bleeding, or passage of the pregnancy before the procedure, and family medicine waiting rooms may not be able to accommodate women experiencing these symptoms.

\section{Aspiration}

Once the cervix is adequately dilated, the appropriate cannula is passed through the cervix so that the 
tip is within the uterus. The MVA syringe is primed by pulling back on the plunger to create a vacuum, and then attached to the cannula. The valve is released and suction curettage can begin. The cannula is rotated and gently moved back and forth, until the smooth sensation of the decidualized endometrium is replaced by a gritty feeling. This indicates that aspiration is complete, and the cannula, tenaculum, and speculum can be removed. The volume of the products of conception increases with gestational age, so that later first trimester procedures may require the MVA syringe to be detached, the contents emptied into a container, and the aspiration repeated. In accordance with the no-touch technique described above, if the cannula is removed it should be handled by the base (where it attaches to the syringe) because the tip must not touch anything unsterile until the procedure is complete.

\section{Inspection of the Aspirate}

After the procedure, the aspirate should be rinsed through a sieve with cold water and suspended in a wide glass dish to facilitate inspection. The gestational sac should be identified, and after 9 weeks fetal parts may also be seen (limbs, calvarium, and spine). A backlight underneath the dish helps. After 10 weeks gestation, all fetal parts should be identified. If there is concern that the procedure may not be complete, an ultrasound should be performed and the uterus reaspirated as indicated (see Management of Common Problems, below).

\section{Postprocedure Care}

Once the provider is confident that the procedure is complete, the patient should have her vital signs, vaginal bleeding, and pain level re-evaluated. Most women are stable and able to get dressed within 5 minutes if they have not received general anesthesia or conscious sedation. Contraception should be started the same day. Intrauterine devices may be placed immediately after the abortion, before the speculum is removed.

\section{Complications}

The minor complications reported in a large review of 170,000 procedural abortions performed at Planned Parenthood clinics ${ }^{24}$ included mild infection (incidence, $0.46 \%$ ); need for re-aspiration (incidence, $0.18 \%$ ); and cervical tear (incidence,
$0.01 \%)$. The overall incidence of these complications was reported as $0.846 \%$.

Complications requiring hospitalization were very rare but included incomplete abortion (incidence, $0.028 \%$ ); sepsis (incidence, $0.021 \%$ ); uterine perforation (incidence, $0.009 \%$ ); and vaginal bleeding (incidence, $0.007 \%$ ). The overall incidence of these complications was $0.071 \%$.

In this review the procedures were performed using an electric vacuum machine and included patients with pregnancies up to 14 weeks gestational age. A recent meta-analysis examined the complication rates of EVA versus MVA for firsttrimester procedural abortion and concluded that MVA may be marginally safer. ${ }^{25}$ In addition, complication rates are increased by including patients with pregnancies at 12 to 14 weeks gestation. For example, all 12 cases of postabortion vaginal bleeding requiring hospitalization occurred in this gestational age range.

A review of 1769 MVA abortions of pregnancies up to 10 weeks gestation performed in a family medicine clinic $^{3}$ reported uterine perforation (incidence, $0.05 \%$ ); pelvic infection (incidence, $0.7 \%$ ); and retained products of conception (incidence, $0.5 \%$ ) as the only complications. None of these cases required hospitalization.

Uterine perforation may be alarming for some providers and clinical protocols should be in place for its detection and management (such as those produced by the National Abortion Federation ${ }^{16}$ ). However, in general, perforation in the first trimester resolves spontaneously and has no sequelae. The risk of death from a first-trimester surgical abortion is less than 1 in 100,000 — significantly less than the risk associated with a pregnancy resulting in a live birth, which varies from 9.8 in 100,000 for the total population to 22.3 in 100,000 for nonHispanic black women. ${ }^{26}$

\section{Management of Common Problems}

Inadequate aspirate, retained products of conception, endometritis, and heavy bleeding represent the majority of problems encountered in first-trimester procedural abortion.

\section{Inadequate Aspirate}

When the aspirated tissue does not appear consistent with gestational age, an ultrasound should be performed to determine whether there is a persis- 
tent gestational sac present in the uterus. If there is, re-aspiration should be performed. A provider may choose to do this with simultaneous or immediate postprocedure ultrasound imaging to be sure that the procedure is complete after the re-aspiration. If the tissue obtained during re-aspiration is still inadequate, the provider may decide to follow serum quantitative beta-human chorionic gonadotropin levels, which should fall during the following 48 hours if the procedure is complete.

\section{Pelvic Infection}

Endometritis after surgical abortion is reduced by approximately $50 \%$ with the routine use of a prophylactic antibiotic such as doxycycline. ${ }^{27}$ If patients are properly counseled regarding the symptoms of pelvic infection, they will usually present early enough to be treated with broader-spectrum oral antibiotics in the outpatient setting. An ultrasound should be performed to look for retained products of conception and, if they are present, aspiration should be performed. Patients with evidence of systemic involvement should be admitted to the hospital for intravenous antibiotics and monitoring.

\section{Heavy Bleeding}

Heavy bleeding occurring during or after the abortion is most commonly caused by cervical laceration or uterine atony. The clinician should inspect the cervix and manage lacerations appropriately. Ring forceps can be used to compress the cervix at the lateral fornices where the cervical blood vessels are located. Rarely, a laceration may require placement of a suture for hemostasis. If the bleeding is thought to be from the uterus, manual uterine compression should be performed and uterotonics, such as methergine, used as needed. ${ }^{16}$

\section{Follow-Up}

Provided women are educated about concerning symptoms, the traditional 2-week follow-up appointment may not be necessary because it does not help to detect complications. ${ }^{11}$ Contraception should be initiated on the day of the procedure. However, it is reasonable to offer patients a follow-up appointment to provide further counseling, preventive care, and answer questions about their chosen method of contraception. Primary care doctors are in an ideal position to use this meeting to address the patient's health more holistically and provide further emotional support. Telephone follow-up may be a reasonable alternative.

\section{Limitations for Family Physicians}

There are potential barriers to the provision of abortion services in a primary care office. An abstract presented at the meeting of the Association of Reproductive Health Professionals identified the following examples: obtaining malpractice coverage; reimbursement for services; staff concerns; purchasing equipment and medications; and the feeling among recent graduates that their skills were not adequate to allow them to practice in a setting where they were the only trained provider. ${ }^{28}$ Support from experienced abortion providers in family medicine, as well as obstetrics and gynecology and other specialties, is also important.

\section{Discussion}

MVA for first-trimester abortion is safe and effective. There are several published reports of MVA abortion being successfully incorporated into established primary care practices and family medicine residency training programs. Incorporating MVA abortion care into primary care settings offers improved continuity of care and fosters the goals of the Future of Family Medicine Project, which strives to create a medical home where a "basket of services" is offered to patients. As the authors of this document state, "new model practices strive to meet patient and community needs for integrated care by giving patients what they want and need ... when they want and need it by anticipating patient needs and designing services to meet those needs." 29

Given that abortion is one of the most commonly requested services in medicine, new model family physicians should meet their patients' needs by integrating early abortion into routine care.

\section{References}

1. Guttmacher Institute. In brief: facts on induced abortion in the United States. Available from http:// www.guttmacher.org/pubs/fb_induced_abortion.html. Accessed 29 December 2008.

2. Weitz TA, Foster A, Ellerston C, Grossman D, Stewart FH. Medical and surgical abortion: rethinking the modifiers. Contraception 2004;69:77-8.

3. Westfall JM, Sophocles A, Burggraf H, Ellis S. Man- 
ual vacuum aspiration for first-trimester abortion. Arch Fam Med 1998;7:559-62.

4. Paul M, Nobel K, Goodman S, Lossy P, Moschella JE, Hammer H. Abortion training in three family medicine programs: resident and patient outcomes. Fam Med 2007;39:184-9.

5. Bennett I, Aguirre AC, Burg J, et al. Initiating abortion training in residency programs: issues and obstacles. Fam Med 2006;38:330-5.

6. Wu JP, Bennett I, Levine JP, Aguirre AC, Bellamy S, Fleischman J. The effect of a simple educational intervention on interest in early abortion training among family medicine residents. Contraception 2006;73:613-7.

7. Dehlendorf C, Brahmi D, Engel D, Grumbach K, Joffe C, Gold M. Integrating abortion training into family medicine residency programs. Fam Med 2007;39:337-42.

8. Brahmi D, Dehlendorf C, Engel D, Grumbach K, Joffe C, Gold M. A descriptive analysis of abortion training in family medicine residency programs. Fam Med 2007;39:399-403.

9. Bennett I, Johnson M, Wu JP, et al. A family medicine training collaborative in early abortion. Fam Med 2007;39:164-6.

10. Rubin SE, Godfrey E, Gold M. Patient attitudes toward early abortion services in the family medicine clinic. J Am Board Fam Med 2008;21:162-4.

11. Grossman D, Ellertson C, Grimes DA, Walker D. Routine follow-up visits after first-trimester induced abortion. Obstet Gynecol 2004;103:738-45.

12. Accreditation Council for Graduate Medical Education. Physical medicine and rehabilitation revised common program requirements (page 25). Available from http://www.acgme.org/acWebsite/downloads/ RRC_progReq/340physicalmedicinerehabilitation 07012007.pdf. Accessed 29 December 2008.

13. Atrash HK, Hogue CJ. The effect of pregnancy termination on future reproduction. Baillieres Clin Obstet Gynaecol 1990;4:391-405.

14. Frank PI, McNamee R, Hannford PC, Kay CR, Hirsch S. The effect of induced abortion on subsequent pregnancy outcome. Br J Obstet Gynaecol 1991;98:1015-24.

15. National Cancer Institute. Summary report: early reproductive events and breast cancer workshop. Available from http://www.cancer.gov/cancerinfo/ ere-workshop-report. Accessed 29 December 2008.
16. National Abortion Federation. Clinical policy guidelines 2007. Available from http://www.prochoice. org/pubs_research/publications/downloads/professional _education/cpgs_2007.pdf. Accessed 29 December 2008.

17. Lichtenberg SE, Paul M, Jones H. First trimester surgical abortion practices: a survey of National Abortion Federation members. Contraception 2001; 64:345-52.

18. Wiebe RE, Rawling M. Pain control in abortion. Int J Gynaecol Obstet 1995;50:41-6.

19. Wiebe E, Podhradsky L, Dijak V. The effect of lorazepam on pain and anxiety in abortion. Contraception 2003;67:219-21.

20. Shapiro AG, Cohen H. Auxiliary pain relief during suction curettage. Contraception 1975;11:25-30.

21. Stubblefield PG. Control of pain for women undergoing abortion. Suppl Int J Gynecol Obstet 1989;3: 131-40.

22. Wiebe ER. Comparison of the efficacy of different local anesthetics and techniques of local anesthesia in therapeutic abortions. Am J Obstet Gynecol 1992; 167:131-4.

23. Allen RH, Goldberg AB. Cervical dilation before first trimester surgical abortion. Contraception 2007;76:139-56.

24. Hakim-Elahi E, Tovell HM, Burnhill MS. Complications of first trimester abortion: a report of 170,000 cases. Obstet Gynecol 1990;76:129-35.

25. Wen J, Cai QY, Deng F, Li YP. Manual versus electric vacuum aspiration for first-trimester abortion: a systematic review. BJOG 2008;115:5-13.

26. US Department of Health and Social Services, Maternal and Child Health Bureau; Available at: http:// mchb.hrsa.gov/pages/page_55.htm

27. Snieders MN, Van Vliet HHAAM, Helmerhorst FM, Low NN. Antibiotic prophylaxis for medical and surgical first-trimester induced abortion (protocol). Cochrane Database Syst Rev 2005;2:CD005217.

28. Lesnewski R, Prine L, Maldonado L. Overcoming barriers to offering abortion care in family medicine [abstract]. Presented at the Association of Reproductive Health Professionals annual meeting, Minneapolis, MN, 26-29 September 2007.

29. Martin JC, Avant RF, Bowman MA, et al. The Future of Family Medicine: a collaborative project of the family medicine community. Ann Fam Med 2004;2(Suppl 1):S3-32. 